\title{
Positive and negative aspects of cell technologies in cerebral diseases
}

Keywords: brain trauma; cell technology, diagnostic, intranasal implantation, stem cell, stroke, therapy

Abbreviations: DWI, diffusion weighted imaging; FITC, fluorescein isothiocyanate; MRI, magnetic resonance imaging; MSC, mesenchymal stem cells; PET, positron emission tomography; SC, stem cells; SPECT, single photon emission computed tomography; TBI, traumatic brain injury; WHO, world health organization

\section{Introduction}

Discouraging statistics characterizes low efficiency of diagnostics and treatment of acute and chronic cerebrospinal diseases and initiates search of new, more effective technologies to resolve that socially important issue. ${ }^{1,2}$ About 6 million people die due to stroke each year according to WHO. ${ }^{1}$ Traumatic Brain Injuries (TBI) claim about 10 million lives annually. ${ }^{3}$ Surgical intervention still remains the key method of treatment of such fatal cerebral diseases as stroke, TBI, cerebral aneurysms and neoplasms. Efficiency of surgery increases with the use of robotic devices (da Vinci Robotic System, SpineAssist, Renaissance Robotic Systems), assimilation of high-tech operations (microsurgical, endovascular, stereotaxic interventions etc.), implementation of combined therapy and new rehabilitation techniques. ${ }^{4-6}$ Opportunities of diagnostic procedures in cerebral diseases are also increased through modern diagnostic equipment (MRI Scan, PET scan, SPECT scan, DWI MRI etc.), that is able to enhance early-stage diagnostics, but still has no effect on treatment outcomes. ${ }^{7-9}$ Current situation remains the basis for constant search of new methods of treatment of cerebral diseases. And these methods have been developed lately in the field of cell biology and neurophysiology.

In particular, cell technologies have been adapted to treatment of socially important diseases in recent years. ${ }^{10}$ There were both followers and opponents of cell technologies' implementation into clinical practice at the early stage, by the way that is usual for any novelty. We will pay attention in terms of observed problem to methods based on stem cells (SC) use in combined treatment of cerebral diseases. SC has been found in various organs and systems of living organism and their role is still not investigated in detail. Three main pools of SC were detected in brain: in the area of olfactory bulbs, in hippocampus and walls of brain ventricles. ${ }^{11}$ Potential of endogenous brain stem cells in health and disease is constantly studied, but oppressive statistics of chronic brain diseases and high mortality due to TBI, stroke, inflammatory and neurodestructive processes speaks for their low effectiveness. There were experimental attempts performed to increase potential of endogenous SC by additional injection of exogenous SC by different ways into the area of pathological focus. The amount of preliminarily in vitro cultivated exogenous stem cells reached thousands and even millions per $1 \mathrm{ml}$. Three ways of administration of SC suspension or mesenchymal SC (MSC) are used in order to implement new method in cerebral diseases: into bloodstream of arterial and venous vessels, into cerebrospinal fluid via lumbar or suboccipital puncture and finally directly into the area of pathological focus in brain after additional craniotomy. ${ }^{12,13}$ Several
Volume 8 Issue 2 - 2018

\author{
Vladimir Kulchitsky, ' Alexandra \\ Zamaro, ' Yuri Shanko, ${ }^{2}$ Stanislav \\ Koulchitsky \\ 'Institute of Physiology, National Academy of Sciences of \\ Belarus, Belarus \\ ${ }^{2}$ Republican Centre of Neurology and Neurosurgery of \\ Ministry of Health, Belarus \\ ${ }^{3}$ Liege University, Belgium
}

Correspondence: Vladimir Kulchitsky, Institute of Physiology, National Academy of Sciences of Belarus, Address: 28 Akademicheskaya Street, Minsk, Belarus, Tel +375 172842458 , Email vladi@fizio.bas-net.by

Received: February 15, 2018 | Published: March 15, 2018

lacks have been stated at the experimental stage of all these ways of $\mathrm{SC}$ administration. Administration of SC into bloodstream - besides distribution in the whole volume of circulating blood-causes problems at the stage of SC penetration through blood-brain barrier. Necessity of additional surgical procedure - craniotomy - also becomes a negative approach for seriously ill patient after stroke or TBI. Administration of SC into cerebrospinal fluid requires measures in order to overcome craniospinal liquor flow and reach destructive area in brain (change of body position, lumbar or suboccipital punctures). First experience of cell technologies use also revealed one more negative effect coming from the risk of SC transformation into tumor cells. ${ }^{14-16}$ Information about malignancy processes usually appears after surgical procedures in cosmetology rooms. However negative response in mass media forms awareness in future patients.

Despite of pronounced side effects, cell technologies demonstrated high effectiveness of reparative potential in cerebral diseases along with minimum of side effects after application of autologous MSC on the area of cranial nerves endings. ${ }^{17,18}$ Olfactory and trigeminal nerves are the most common targets for application in the modelling of brain diseases. ${ }^{17-19}$ For example, intranasal injection of FITC-labeled MSC into the area of olfactory nerve endings resulted in appearance of fluorescent MSC already in 30 minutes in olfactory bulbs and in several hours - in trauma region, mainly in anterior cranial fossa. ${ }^{18,19}$ Somatotropic distribution of stem cells in brain was substantiated by the fact of predominant migration of MSC to destructed brain areas located in posterior cranial fossa after preliminary administration into the area of trigeminal nerve endings. Therefore, the technique of MSC administration based on perineural migration of MSC to neurodestructive region was experimentally developed for clinical application. ${ }^{17-19}$

The next self-evident conclusion should be mentioned: it was experimentally and clinically proved, that autologous stem cells are the safest biomaterial for recovery of violated brain functions. The use 
of autologous SC minimizes risk of tumor development associated with administration of exogenous stem cells into living organism. Experimenter and clinician should follow unbreakable principle "first, do no harm" in order to constantly achieve positive effects of clinical technologies use and level negative effects.

\section{Acknowledgements}

This pooled analysis was funded by OOO Synergy.

\section{Conflict of interest}

All listed authors concur with the submission of the manuscript; all authors have approved the final version. The authors have no financial or personal conflicts of interest.

\section{References}

1. Kelly PJ, Crispino G, Sheehan O, et al. Incidence, event rates, and early outcome of stroke in Dublin, Ireland: the North Dublin population stroke study. Stroke. 2012;43(8):2042-2047.

2. Feigin VL, Norrving B, Mensah GA. Global Burden of Stroke. Circulation Research. 2017;120(3):439-448.

3. Nasser M, Bejjani F, Raad M, et al. Traumatic Brain Injury and Blood-Brain Barrier Cross-Talk. CNS \& Neurological Disorders Drug Targets. 2016;15(9):1030-1044.

4. Onen MP, Naderi S. Robotic Systems in Spine Surgery. Turk Neurosurgery. 2014;24(3):305-311.

5. Mattei TA, Rodriguez AH, Sambhara D, et al. Current state-of-theart and future perspectives of robotic technology in neurosurgery. Neurosurgical Review. 2014;37(3):357-366.

6. Bernardo A. The Changing Face of Technologically Integrated Neurosurgery: Today's High-Tech Operating Room. World Neurosurgery. 2017;106:1001-1014.

7. Hannawi Y, Stevens RD. Mapping the Connectome Following Traumatic Brain Injury. Current Neurology and Neuroscience Reports. 2016;16(5):44.
8. Hojjati M, Badve C, Garg V, et al. Role of FDG-PET/MRI, FDGPET/CT, and Dynamic Susceptibility Contrast Perfusion MRI in Differentiating Radiation Necrosis from Tumor Recurrence in Glioblastomas. J Neuroimaging. 2018;28(1):118-125.

9. Heiss WD, Rosenberg GA, Thiel A, et al. Neuroimaging in vascular cognitive impairment: a state-of-the-art review. BMC Medicine. 2016;14(1):174.

10. Martínez-Morales PL, Revilla A, Ocaña I, et al. Progress in stem cell therapy for major human neurological disorders. Stem Cell Rev. 2013;9(5):685-699.

11. Rueger MA, Androutsellis-Theotokis A. Identifying endogenous neural stem cells in the adult brain in vitro and in vivo: novel approaches. Curr Pharm Des. 2013;19(36):6499-6506.

12. Chen L, Qiu R, Xu Q. Stem cell therapy for ischemic stroke. $J$ Nanosci Nanotechnol. 2014:14(1):976-982.

13. Vu Q, Xie K, Eckert M, et al. Meta-analysis of preclinical studies of mesenchymal stromal cells for ischemic stroke. Neurology. 2014;82(14):1277-1286.

14. Prockop DJ. Defining the probability that a cell therapy will produce a malignancy. Mol Ther. 2010;18 (7):1249-1250.

15. Kuroda T, Yasuda S, Sato Y. Tumorigenicity studies for human pluripotent stem cell-derived products. Biol Pharm Bull. 2013;36(2):189-192.

16. Prockop DJ. The exciting prospects of new therapies with mesenchymal stromal cells. Cytotherapy. 2017;19(1):1-8.

17. Donega V, Nijboer $\mathrm{CH}$, Braccioli L, et al. Intranasal administration of human MSC for ischemic brain injury in the mouse: in vitro and in vivo neuroregenerative functions. PLoS One. 2014;9(11):e112339.

18. Stukach Y, Koulchitsky S, Shanko Y, et al. Targeted migration of stem cells in the model of brain trauma. Proceedings of the seventh workshop on experimental models and methods in biomedical research. Sofia, Bulgaria. 2016;143-145.

19. Stukach YP, Shanko YG, Kulchitsky VA. Experimental substantiation of stem cells delivery to the brain through cerebral nerves endings. Biological motility. 2016;232-235. 